\title{
Editorial: Pathologic Findings in Stranded Marine Mammals: A Global Perspective
}

\author{
Debra L. Miller ${ }^{1 *}$, Alvin C. Camus ${ }^{2}$ and Stephen A. Raverty ${ }^{3}$ \\ ${ }^{1}$ Center for Wildlife Health and One Health Initiative, University of Tennessee, Knoxville, Knoxville, TN, United States, \\ ${ }^{2}$ Department of Pathology, University of Georgia, Athens, GA, United States, ${ }^{3}$ Animal Health Centre, British Columbia \\ Ministry of Agriculture, Abbotsford, BC, Canada
}

Keywords: editorial, marine mammal, one health, stranding, pathology

\author{
Editorial on the Research Topic
}

Pathologic Findings in Stranded Marine Mammals: A Global Perspective

It has been 2 years since Greg Bossart reached out to the marine mammal community asking colleagues, collaborators, and graduate students to provide a series of original research and review articles of the gross, histopathologic, and clinicopathologic findings of stranded marine mammals from a global research perspective. With the publications of the third edition of the CRC Handbook of Marine Mammal Medicine and Pathology of Wildlife and Zoo Animals in 2018, Greg wanted to be especially vigilant about securing manuscripts describing more recent and novel disease entities which may not have been captured in either textbook and extended invitations to people and stranding response programs that historically may have been underrepresented in the marine mammal stranding and pathology literature. His intent was to integrate pathologic findings from a global (i.e., rather than regional or local) perspective in a single Research Topic format that would provide context for population health and sustainability. In turn, this collection of papers would establish baseline information on emerging or re-surging disease patterns that may be of value to marine biologists, research scientists, clinicians, epidemiologists, pathologists, and other marine mammal specialists. We further hope that this Research Topic will inform managers involved with implementing or reviewing conservation policies. A lot has transpired in the last 2 years, including the unfortunate and untimely death of our friend and colleague, Greg, as well as the global COVID pandemic. When Greg asked us to help him assemble this collection, we were hoping that he would be here to see it to fruition, so his death has left us at a loss as it did so many others. Nevertheless, here we are 2 years later, and Greg's dream is realized and surpassed due to the amazing members and contributions from the marine mammal community.

We all are aware that marine mammal strandings (mass or single animal) are global events and have been reported as far back as Aristotle's time; however, Unusual Mortality Events (UMEs) (i.e., marine mammal strandings, which occur at significantly elevated rates, temporal or geographic change in strandings, potential significant morbidity and mortality in a population, and other criteria) are now occurring at an increasing rate, often with no conclusive cause of strandings or deaths. These UME's also afford a unique opportunity to further document the natural history and anatomic and physiologic adaptations of these species as well as characterize pathologic findings that may contribute directly or indirectly to suboptimal health and stranding events. Unfortunately, in some cases (i.e., mass stranding events) the cause(s) of the stranding are enigmatic with little or no associated pathology that would suggest a reason for the stranding. The advancement and application of novel diagnostic and imaging techniques detailed within this series of papers has significantly improved our ability to better define specific disease entities.

Miller DL, Camus AC and Raverty SA (2021) Editorial: Pathologic Findings in Stranded Marine Mammals: A Global 
Importantly, many of the diseases now characterized from marine mammal strandings involve emerging pathogens that may cycle exclusively in the marine environment or represent terrestrially sourced pathogens (pollutagens) and have important population and environmental health significance. Pattern recognition or morphologic diagnosis of disease events will no longer suffice to understand potential implications of novel clinical or pathologic findings. Greg has long advocated a problem-oriented approach with more unusual or exotic diagnostic findings.

Gross and histopathologic findings remains the gold standard for characterizing the morphologic features of disease and linking an etiology to the pathology, cause of death and possibly, implementing appropriate management or mitigating options. Likewise, marine mammal clinical pathology parameters are increasingly documented and validated from both stranded marine mammals and free-ranging marine mammal health assessments and their values and reference ranges are being incorporated into formulating prognoses, informing treatments and deciding disposition of rehab animals (Ewing et al.). Ultimately, our understanding of the pathologic basis of disease and causes of mortality in stranded marine mammals can serve as the first step in evaluating complex and dynamic marine ecosystem health. Indeed, recent studies have demonstrated that marine mammals are important sentinel animals that reflect environmental health concerns and parallel emerging public health issues, all of which embraces a One Health approach. A glimpse within the pages of this Research Topic (Pathologic Findings in Stranded Marine Mammals: A Global Perspective), highlights the disparate discoveries of the marine mammal community within a One Health context.

There were 28 manuscripts accepted for this special issue of Frontiers in Marine Sciences, including 16 from the United States: 4 from California, 3 from Alaska, 2 each from the Pacific Northwest, Northeast, Southeast, and span the western seaboard of the United States. There were 6 papers from Europe, consisting of 3 from the Canary Islands, 2 from the North Sea Region and 1 from France, 2 from South America, including 1 from Brazil and the other from Chile, 2 from Southeast Asia, 1 each from Hong Kong and Japan and 2 from Canada. The target species comprised 19 cetaceans including 13 papers describing findings in multiple species and 6 studies which detailed findings in individual species, focusing on beluga, rough toothed dolphins, harbor porpoises, bottlenose dolphins, gray whales and a Bryde's whale. Reviews are presented on case series of aggression by conspecifics, differentials for infectious meningoencephalitis, harbor porpoise specific pathologic findings, the effects of acute entrapment, and models of decompression sickness. The novel use postmortem CT and MR imaging to better define pathology, particularly in autolyzed carcasses and a postmortem scenario that may account for cetacean sinking to depth, bloat and refloating are provided. These have important implications for back casting in mass stranding events as well as with catastrophes, such as with oil spills. Novel approaches to understand the effects of anthropogenic noise on cetaceans and well as development of a laboratory model of gas bubble disease are described.

There were 5 reports on sea otters including major disease reviews in California and Alaska as well as emerging individual animal and population level health concerns with harmful algal toxins, Helicobacter associated gastric ulcers and a zoonotic pathogen, capillariasis. There were 2 papers describing novel viral pathogens (picornavirus and orthoreovirus) in harbor seals stranded in the north eastern Pacific and an overview of pathologic findings in a harbor seal population at carrying capacity. A single paper described the complex interplay of contaminants, herpesvirus infection, and population genetics with the emergence of urogenital carcinoma in California sea lions. Although disease entities appear unique to specific marine mammal species, there is occasional overlap and involvement of new species not typically associated with specific pathogens, environmental insults, or other disorders.

Marine mammal stranding events continue to be investigations that capitalize on mystery with a "who done it" plot. Moore provides us with an overview of the various outcomes that might be encountered in any one stranding event, and what clues might be offered regarding factors contributing to mortality and death. Many of these events are reported within the pages of this collection. Sometimes, such as in the report by Alvarado-Rybak et al. the geographic ruggedness of the coastline makes documentation an unsurmountable challenge, yet these authors provide a glimpse into the causes of deaths within one of these more challenging areas to access. Other reports focus on events happening over a span of time within a particular geographic region. Examples include the report by Schick et al. on findings in two cetacean species over a 20 year period in the south-eastern North Sea, and Miller et al. on southern sea otters (Enhydra lutis nereis) over a 14 year period. In contrast, the report by Ewing et al. focuses on events that occurred at a single point in time (i.e., the 2005 Marathon Key stranding). An interesting report is by Ashley et al. who discuss the causes of mortality in a population at carrying capacity. Similarly, Stimmelmayr and Gull and review the current state of the "recovered" eastern North Pacific gray whale (Eschrichitius robustus) in light of urbanization and rapidly changing habitat. Both of these studies offer insight that can be applied to current and future management plans.

Infectious agents are often the cause of death, no matter the host species, and although they may play a primary role in disease and death, they may remain subclinical or opportunistic, becoming a problem only in hosts with weakened health. One example is Helicobacter, and as reported by Batac et al. Helicobacter-induced gastric ulcers are a contributor in morbidity and mortality in southern sea otters (Enhydra lutris nereis). Regardless of being primary or opportunistic, infectious agents seem to be constantly evolving with novel organisms often appearing on the scene. For example, Nielsen et al. report a novel Orthoreovirus from harbor seals (Phoca vituline) that stranded in Puget Sound over the course of 1 year, and Rodrigues et al. report novel Aquamaviruses from 
ribbon (Histriophoca fasciata) and harbor seals in Puget Sound within that same time period. Long-term studies can also be important in identifying trends in morbidity and mortality due to particular pathogens. For example, Huntington et al. conducted an epidemiological analysis of northern sea otters (Enhydra lutris kenyoni) necropsied over a span of 10 years and found a pattern in geographic location and the diagnosis of Strep syndrome that they suggest may be linked to increasing environmental temperatures. Similarly, environmental stressors are suspected for an increased incidence of fungal infections and reported by Huggins et al. in the Pacific northwest and Kapetanou et al. along the Dutch coastline. Parasites are included as infectious agents and Sierra et al. provide an excellent study on brain pathogens in cetaceans, including Morbillivirus, Herpesvirus, Toxoplasma gondii, Brucella sp., and Nasitrema sp. These pathogens are deadly, especially when targeting the brain. Toxoplasma is especially perplexing as this protozoan has a terrestrial species as its definitive host, yet it wreaks havoc on species throughout the marine environment. Diaz-Delgado et al. provide a case report of fatal systemic toxoplasmosis in a Bryde's whale (Balaenoptera edeni) by a novel $T$ gondii. This is not the only zoonotic parasite covered. Miller et al. report on hepatitis caused by Capillaria hepatica, a parasite generally associated with close proximity to rats, in southern sea otters (Enhydra lutris nereis), which is a coastal dwelling species. Interestingly, species variation in tissue reaction and immune response of hosts can impact histochemical and immunohistochemical results for some parasites such as reported for hepatic trematodiasis in odontocetes by Nakagun and Kobayashi. Like so many other of the studies highlighted, these discoveries have implications for human health as well as predator species and overall marine mammal health.

Contaminants and biologic toxins are increasingly a concern for all life on earth and unfortunately the marine environment not been spared. In some geographic regions, exposed marine mammals are especially impacted. Miller et al. provide a detailed gross and histopathologic depiction of lesions associated with domoic acid toxicosis throughout the course of the disease. Of course, humans contribute to environmental contamination on a daily basis, including products we consume or put on our bodies, products we use, or industrial production, just to name a few. Page-Karjian et al. provide a summary of anthropogenic contaminant and pathologic trends in cetaceans that shed light on potential contributors to declining health in cetaceans. With heavy metals, mercury has often been studied because of its bioaccumulation and biomagnification within the food chain, and because oceans can act as a sink. With climate change in the arctic, increasing release of mercury associated with melting permafrost may have profound impacts on marine mammal health and hunter communities with regards to food safety and security. Lian et al. provide an interesting look at how skin biopsies collected from live cetaceans can be used to assess contaminant load. In some cases, the role of the contaminant is not entirely clear. For example, Gulland et al. report that $18-23 \%$ of adult California sea lions (Zalophus californianus) necropsied over a span of 40 yrs had urogenital carcinoma, and provide support of a link between organochlorines, Otarine herpesvirus-1 and development of neoplasia.

Boat (vessel) strike and other anthropogenic activities profoundly impact the status of marine mammal populations worldwide. Cumulatively, it is one of the most significant contributors to population decline. Novel clinical and pathologic presentations of trauma are included in this collection. One example includes trauma associated with intra and interspecies aggression. Puig-Lozano et al. review findings of encounters over a span of 18 years and provide gross and microscopic findings of the lesions to better define this entity. Epple et al. present two newly identified traumatic lesions in dolphin abdomens putatively linked to peracute underwater entrapment in fishing gear. Similarly, Velazquez-Wallraf et al. provide new insights into decompressive pathology observed in cetaceans gained through use of an experimental animal (rabbit) model.

The contributors to this issue have provided disparate and amazing information beyond the more conventional stranding or pathogen specific information. Opportunities have been pursued to glean as much additional information in individual cases or case series as possible. This information moves us forward in our approach to data collection and our understanding of some of the unique attributes of these amazing creatures. For example, Morell et al. identified a common membrane motor protein (prestin) expressed by both whales and bats, further linking it to echolocation. This study, along with another by Morell et al. advances our identification of hearing loss in cetaceans. Similarly, Dabin et al. explored cause and effects of formation of vaginal calculi in dolphins, a condition that afflicts many mammals, including humans. Finally, Kot et al. present nascent approaches to use imaging tools postmortem to overcome obstacles of decomposition when trying to diagnose cause of death in stranded cetaceans.

This amazing compilation can be held up as a signal/warning/beacon for "One Health". We only need to look at investigations into the root causes of many of these pathologies and see the human hand at work. As Greg echoed over the course of his career and lifetime, we must continue to be vigilant and recognize the valuable role marine mammals serve as sentinels for human health. Greg's philosophy was to provide solid case definitions and baseline information on the health of stranded and in some cases, live caught marine mammals with the intent that this information may be of future value with the emergence of new disease entities. The work compiled in this special issue further substantiates Greg's vision and, in some cases significantly advances the scientific understand of more complex and dynamic ecologic and pathologic processes. We know that Greg would have been humbled and deeply moved by the contribution of so many people from around the world and in such a variety of specialties. We thank the authors for their 
irredeemable support and kindness through the review and publication process.

\section{AUTHOR CONTRIBUTIONS}

All authors listed have made a substantial, direct, and intellectual contribution to the work and approved it for publication.

Conflict of Interest: The authors declare that the research was conducted in the absence of any commercial or financial relationships that could be construed as a potential conflict of interest.
Publisher's Note: All claims expressed in this article are solely those of the authors and do not necessarily represent those of their affiliated organizations, or those of the publisher, the editors and the reviewers. Any product that may be evaluated in this article, or claim that may be made by its manufacturer, is not guaranteed or endorsed by the publisher.

Copyright (c) 2021 Miller, Camus and Raverty. This is an open-access article distributed under the terms of the Creative Commons Attribution License (CC BY). The use, distribution or reproduction in other forums is permitted, provided the original author(s) and the copyright owner(s) are credited and that the original publication in this journal is cited, in accordance with accepted academic practice. No use, distribution or reproduction is permitted which does not comply with these terms. 